\title{
Low uric acid level increases the risk of infectious mononucleosis and this effect is more pronounced in women
}

\author{
LI ZHANG $^{1 *}$, PINGPING ZHOU ${ }^{2 *}$, ZHAOWEI MENG ${ }^{2}, \mathrm{LU} \mathrm{GONG}^{1}, \mathrm{CHONGJIE} \mathrm{PANG}^{1}, \mathrm{XUE} \mathrm{LI}^{2}$,

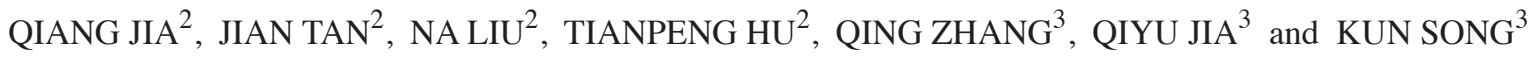 \\ Departments of ${ }^{1}$ Infectious Diseases, ${ }^{2}$ Nuclear Medicine and ${ }^{3}$ Health Management, \\ Tianjin Medical University General Hospital, Tianjin 300052, P.R. China
}

Received April 13, 2017; Accepted September 28, 2017

DOI: $10.3892 / \mathrm{mco} .2017 .1433$

\begin{abstract}
Infectious mononucleosis (IM) due to Epstein-Barr virus infection is common. Uric acid (UA) is an important endogenous antioxidant. To the best of our knowledge, the association between UA and IM has not been comprehensively investigated to date. The aim of the present study was to investigate this association in Chinese patients. A total of 95 patients (47 men and 48 women) with IM were recruited, along with 95 healthy controls. Clinical data were classified by patient sex. Receiver operating characteristic (ROC) curve analysis was adopted to determine the cut-off values of UA for IM diagnosis and prediction. Crude and adjusted odds ratios (ORs) of UA for IM were analyzed by binary logistic regression. The UA levels were significantly lower in IM patients compared with those in controls. In addition, UA levels in men were significantly higher compared with those in women. The ROC curve demonstrated good diagnostic and predictive values of UA for IM in both sexes. The UA cut-off values were 326.00 and $243.50 \mu \mathrm{mol} / 1$ for diagnosing IM in men and women, respectively, with a diagnostic accuracy of 76.596 and $80.208 \%$, respectively. Binary logistic regression analysis revealed a significant risk of IM in the low UA quartiles in both sexes. Following adjustments, the ORs even increased. Women with low UA levels appeared to be more susceptible to IM. For example, the crude ORs in quartile 1 were 24.000 and 52.500 for men and women, respectively, and the respective adjusted ORs were 31.437 and 301.746 (all $\mathrm{P}<0.01)$. To the best of our knowledge, the present study is the first to demonstrate the inverse association between UA and IM, suggesting a progressive decrease of antioxidant reserve in IM. Moreover, low UA was suggestive of IM, particularly in women.
\end{abstract}

Correspondence to: Professor Zhaowei Meng, Department of Nuclear Medicine, Tianjin Medical University General Hospital, 154 Anshan Road, Heping, Tianjin 300052, P.R. China

E-mail: jamesmencius@163.com

*Contributed equally

Key words: infectious mononucleosis, Epstein-Barr virus, uric acid, sex

\section{Introduction}

Infectious mononucleosis (IM) is an important clinical entity that is associated with Epstein-Barr virus (EBV) infection $(1,2)$. This clinical manifestation was first described in 1889, but the term IM was coined in 1920, when it was discovered that a number of patients with glandular fever had similar blood films (3). In 1968, the then newly discovered EBV was identified as the cause of IM (4). The currently estimated incidence of IM is at $\sim 500$ cases per 100,000 persons annually. IM diagnosis is often established with the classical clinical triad of pharyngitis, fever and lymphadenopathy. Serological testing for the identification of EBV antibodies is required for a definitive diagnosis $(1,2)$. The treatment of patients with IM is mainly supportive. Corticosteroids are considered as the standard treatment for severe complications associated with $\operatorname{IM}(1,2)$.

Uric acid (UA) is a purine degradation metabolite. A high serum level of UA is considered harmful. Hyperuricemia is considered to be closely associated with a number of metabolic disorders (5-7). For example, it was previously demonstrated that UA and metabolic syndrome were closely associated, and young women with hyperuricemia were at the highest risk of developing metabolic syndrome (5). Our recent study investigated subclinical thyroid dysfunction and hyperuricemia. It was demonstrated that, in subjects with hyperuricemia, mild hypothyroidism was a risk factor for men, while not for women (6). UA is also an important endogenous antioxidant, as well as a natural scavenger of peroxynitrate. Abnormalities in the serum levels of UA have been observed in several diseases. For example, a low UA level has been detected in stroke (8-10), multiple sclerosis (MS) $(11,12)$, infections of the central nervous system (CNS) $(13,14)$ and leprosy reaction episodes $(15)$. As regards IM, the number of previous related studies is limited and the results are conflicting. A total of three early articles (16-18) with small number of recruited subjects and one previous case report (19) were retrieved. Dylewski et al (16) investigated 35 cases with IM after a case report, and reported that 7 men and 2 women had UA levels above the laboratory's upper limit of normal. Cowdrey (17) reported UA elevation during the first 10 days of the disease course in 21 patients. However, Sugita et al (19) described a case of a 27-month-old boy with persistent EBV infection and CNS manifestations, who had lymphadenopathy and low UA levels. 
Therefore, the aim of the present study was to analyze the associations between UA and IM in a comprehensive manner, in order to determine whether low UA is a significant risk factor for IM, and whether there is a sex difference.

\section{Patients and methods}

Patients. The present study was conducted under collaboration between the Departments of Infectious Diseases, Nuclear Medicine and Health Management of Tianjin Medical University General Hospital (Tianjin, China). Between December 2014 and December 2015, a total of 95 patients (47 men and 48 women) with a confirmed diagnosis of IM were recruited. All the patients were admitted to the Department of Infectious Diseases of our hospital.

Controls. Between June 2015 and September 2015, 95 healthy subjects (47 men and 48 women) were enrolled in the normal control cohort from the Department of Health Management of our hospital. The control subjects visited our institution to receive a routine annual health checkup.

Ethics. The Institutional Review Board of Tianjin Medical University General Hospital approved the ethical and methodological aspects of the study protocol and all the participants provided written informed consent. All the methods were performed in accordance with the relevant ethical regulations.

Parameter measurements. For patients with IM, blood tests and anthropometric measurements were performed upon admission to the Department of Infectious Diseases. For the healthy controls, blood tests and anthropometric measurements were performed upon visiting our institution.

Physical examination included body height $(\mathrm{BH})$ and body weight (BW) measurement. Body mass index (BMI) was calculated as BW divided by BH squared $\left(\mathrm{kg} / \mathrm{m}^{2}\right)$. Fasting blood tests were performed following venipuncture, and serological parameters were measured.

White blood cell (WBC) count, red blood cell (RBC) count, hemoglobin $(\mathrm{Hb})$ level and platelet (PLT) count were measured using a hemocytometer (Sysmex Corporation, Kobe, Japan). Alanine aminotransferase (ALT), aspartate aminotransferase (AST), total bilirubin (TB), blood urea nitrogen (BUN), creatinine $(\mathrm{Cr})$ and UA were enzymatically determined by an auto-analyzer (model 7170; Hitachi, Tokyo, Japan).

Antibodies (IgM and IgG) against specific EBV antigens were measured by the enzyme-linked immunosorbent assay method using a commercial kit (Euroimmun; Medizinische Labordiagnostika AG, Lübeck, Germany).

Diagnostic criteria. The diagnosis of IM was generally based on the clinical presentation, the presence of atypical lymphocytes on a peripheral blood smear, and a positive heterophile antibody test. Serological testing for the identification of antibodies against specific EBV antigens was required in order to establish a definitive diagnosis $(1,2)$. Hyperuricemia was defined as UA $>420 \mu \mathrm{mol} / \mathrm{l}$ in men and $>360 \mu \mathrm{mol} / 1$ in women (5).

Statistical analysis. All data are presented as mean \pm standard deviation; men and women were separately analyzed.
Differences in indices between the two groups of patients were measured by the independent samples t-test. The Chi-squared test was used to compare differences in prevalence. Pearson's bivariate correlation was used to assess the correlation between UA and other variables. Receiver operating characteristic (ROC) curves were drawn and diagnostic efficacies were then determined. After the optimal cut-off UA value was selected, the sensitivity, specificity, diagnostic accuracy, positive predictive value and negative predictive value for differential diagnosis were assessed. By stratifying data with UA quartiles, odds ratio (OR) for IM with $95 \%$ confidence interval (CI) was calculated by binary logistic regression models. SPSS version 17.0 (SPSS Inc., Chicago, IL, USA) was used to conduct statistical analyses and significance was set at $\mathrm{P}<0.05$.

\section{Results}

Characteristics of the participants. The measured variables were separately compared in men and women (Tables I and II). In men, WBC count, ALT and AST were significantly higher in patients with IM compared with control subjects, whereas $\mathrm{RBC}$ count, $\mathrm{Hb}$ and UA levels were significantly lower in patients with IM compared with control subjects. In women, ALT and AST were significantly higher in IM patients, whereas RBC count, $\mathrm{Hb}, \mathrm{TB}, \mathrm{BUN}, \mathrm{Cr}$ and UA levels were significantly lower in IM patients compared with controls.

UA differences between sexess. Comparison of UA levels between sexes in IM patients revealed significantly higher levels in men $(\mathrm{t}=5.056, \mathrm{P}<0.01)$. Similarly, comparison of UA levels between sexes in control subjects also revealed significantly higher levels in men $(\mathrm{t}=7.531, \mathrm{P}<0.01)$. There was a lower incidence of hyperuricemia in men with IM, but the difference was not statistically significant. However, no women with IM had hyperuricemia, which was statistically significantly different from the control group (Table III).

Correlations between key variables. Correlation coefficients between UA and other variables were calculated to determine whether there were any significant associations (Table IV). Statistically significant positive correlations were found between UA and BMI, RBC count, $\mathrm{Hb}, \mathrm{TB}, \mathrm{BUN}$ and $\mathrm{Cr}$ in men. In women, UA was statistically significantly positively correlated with RBC count, $\mathrm{Hb}, \mathrm{BUN}$ and $\mathrm{Cr}$.

Diagnostic and predictive values of UA for IM. Based on the ROC analysis, UA demonstrated good diagnostic and predictive values for IM (Fig. 1). The cut-off values were calculated as 326.00 and $243.50 \mu \mathrm{mol} / 1$ in men and women, respectively, with area under the curve values of 0.809 and 0.835 , respectively (both $\mathrm{P}<0.01$ ). The sensitivity, specificity, diagnostic accuracy, positive predictive value and negative predictive value were found to be $74.500,78.700,76.596,75.510$ and $77.778 \%$, respectively, for men, while the respective values for women were $75.000,85.400,80.208,77.358$ and $83.721 \%$.

Risk of IM in different UA quartiles. Binary logistic regression models were used to calculate the risk of IM in the two sexes (Table V). Crude OR calculation was performed with $\mathrm{UA}$ in the highest quartile as reference, and significant risk 
Table I. Parameter characteristics in men.

\begin{tabular}{|c|c|c|c|}
\hline Parameters & Patients with IM & Controls & P-value \\
\hline Number of subjects & 47 & 47 & \\
\hline Age (years) & $37.40 \pm 16.83$ & $37.68 \pm 16.83$ & -0.080 \\
\hline BMI $\left(\mathrm{kg} / \mathrm{m}^{2}\right)$ & $23.58 \pm 3.02$ & $24.74 \pm 3.50$ & -1.725 \\
\hline WBC $\left(\times 10^{9} / 1\right)$ & $7.17 \pm 3.81$ & $5.93 \pm 1.49$ & $2.085^{\mathrm{a}}$ \\
\hline $\operatorname{RBC}\left(\times 10^{12} / 1\right)$ & $4.46 \pm 0.51$ & $5.19 \pm 0.38$ & $-7.870^{\mathrm{b}}$ \\
\hline $\mathrm{Hb}(\mathrm{g} / \mathrm{l})$ & $133.19 \pm 14.59$ & $155.15 \pm 10.21$ & $-8.453^{b}$ \\
\hline PLT (x 109/1) & $208.47 \pm 80.43$ & $214.81 \pm 46.92$ & -0.467 \\
\hline $\operatorname{ALT}(\mathrm{U} / \mathrm{l})$ & $86.72 \pm 108.67$ & $27.57 \pm 29.00$ & $3.605^{\mathrm{b}}$ \\
\hline AST (U/l) & $55.53 \pm 76.95$ & $24.45 \pm 30.67$ & $2.573^{\mathrm{a}}$ \\
\hline $\mathrm{TB}(\mu \mathrm{mol} / \mathrm{l})$ & $12.24 \pm 11.86$ & $14.12 \pm 6.62$ & -0.952 \\
\hline BUN (mmol/l) & $4.10 \pm 1.74$ & $4.56 \pm 1.22$ & -1.496 \\
\hline $\mathrm{Cr}(\mu \mathrm{mol} / \mathrm{l})$ & $76.30 \pm 28.92$ & $83.34 \pm 12.75$ & -1.528 \\
\hline $\mathrm{UA}(\mu \mathrm{mol} / \mathrm{l})$ & $278.98 \pm 96.58$ & $373.00 \pm 72.49$ & $-5.338^{b}$ \\
\hline \multicolumn{4}{|c|}{$\begin{array}{l}\text { a } \mathrm{P}<0.05 \text {. }{ }^{\text {b }}<0.01 \text { (independent samples t-test). Values are presented as } \\
\text { mean } \pm \text { standard deviation. IM, infectious mononucleosis; BMI, body } \\
\text { mass index; WBC, white blood cell; RBC, red blood cell; Hb, hemo- } \\
\text { globin; PLT, platelet; ALT, alanine aminotransferase; AST, aspartate } \\
\text { aminotransferase; TB, total bilirubin; BUN, blood urea nitrogen; Cr, } \\
\text { creatinine; UA, uric acid. }\end{array}$} \\
\hline
\end{tabular}

was demonstrated for IM in quartile 1 and 2 for both sexes. Adjusted OR calculation included age and BMI as covariates. A significantly enhanced risk for IM was displayed in quartile 1 and 2 for both sexes. Of note, women with low serum UA appeared to be more susceptible to IM. The crude ORs in quartile 1 were 24.000 (95\% CI: 4.381-131.472) and 52.500 (95\% CI: 8.640-319.028) for men and women, and the adjusted ORs were 31.437 (95\% CI: 4.680-211.181) and 301.746 (95\% CI: 25.160-3618.861), respectively (all $\mathrm{P}<0.01$ ).

\section{Discussion}

The aim of the present study was to investigate whether UA has diagnostic and predictive value for IM, prompted by the fact that a low UA level was found to be associated with pathological conditions such as stroke $(8-10)$, MS $(11,12,20)$ and CNS infections $(13,14)$. Our research group previously investigated UA, but the focus was the association of hyperuricemia with various metabolic disorders (5-7). The fact that low UA levels have important clinical implications has become intriguing; therefore, collaborative efforts were focused on investigating the association between UA and IM. It was demonstrated that UA was significantly lower in patients with IM compared with healthy controls. Low UA level was found to have adequate diagnostic and predictive power for IM. Subjects with low UA levels, indicating low antioxidant reserve, were significantly more likely to develop IM, and these effects were more pronounced in women.

IM commonly affects patients who have had a primary EBV infection during childhood or adolescence. As the overall socioeconomic and sanitary conditions have improved, EBV infection in early childhood has become less common (1), with no obvious annual cycles or seasonal changes in incidence, and
Table II. Parameter characteristics in women.

\begin{tabular}{lccc}
\hline Parameters & Patients with IM & Controls & P-value \\
\hline Number of subjects & 48 & 48 & \\
Age $($ years $)$ & $41.27 \pm 17.54$ & $41.19 \pm 17.35$ & 0.023 \\
BMI $\left(\mathrm{kg} / \mathrm{m}^{2}\right)$ & $23.52 \pm 3.81$ & $22.60 \pm 2.94$ & 1.314 \\
WBC $\left(\mathrm{x} 10^{9} / \mathrm{l}\right)$ & $6.29 \pm 3.62$ & $5.17 \pm 1.54$ & 1.975 \\
RBC $\left(\mathrm{x} 10^{12} / \mathrm{l}\right)$ & $3.93 \pm 0.43$ & $4.44 \pm 0.27$ & $-6.954^{\mathrm{a}}$ \\
$\mathrm{Hb}(\mathrm{g} / \mathrm{l})$ & $113.54 \pm 13.34$ & $130.73 \pm 8.49$ & $-7.530^{\mathrm{a}}$ \\
$\mathrm{PLT}\left(\mathrm{x} 10^{9} / \mathrm{l}\right)$ & $235.65 \pm 90.10$ & $226.15 \pm 50.15$ & 0.638 \\
$\mathrm{ALT}(\mathrm{U} / \mathrm{l})$ & $61.35 \pm 103.51$ & $14.19 \pm 6.84$ & $3.150^{\mathrm{a}}$ \\
$\mathrm{AST}(\mathrm{U} / \mathrm{l})$ & $45.60 \pm 58.11$ & $16.68 \pm 6.08$ & $3.393^{\mathrm{a}}$ \\
$\mathrm{TB}(\mu \mathrm{mol} / \mathrm{l})$ & $7.63 \pm 5.42$ & $11.03 \pm 6.01$ & $-2.913^{\mathrm{a}}$ \\
$\mathrm{BUN}(\mathrm{mmol} / \mathrm{l})$ & $2.97 \pm 1.05$ & $4.15 \pm 1.15$ & $-5.242^{\mathrm{a}}$ \\
$\mathrm{Cr}(\mu \mathrm{mol} / \mathrm{l})$ & $52.13 \pm 9.46$ & $60.77 \pm 9.59$ & $-4.446^{\mathrm{a}}$ \\
$\mathrm{UA}(\mu \mathrm{mol} / \mathrm{l})$ & $195.27 \pm 61.25$ & $272.75 \pm 56.41$ & $-6.446^{\mathrm{a}}$
\end{tabular}

${ }^{\text {a }} \mathrm{P}<0.01$ (independent samples $\mathrm{t}$-test). Values are presented as mean \pm standard deviation. IM, infectious mononucleosis; BMI, body mass index; WBC, white blood cell; RBC, red blood cell; Hb, hemoglobin; PLT, platelet; ALT, alanine aminotransferase; AST, aspartate aminotransferase; TB, total bilirubin; BUN, blood urea nitrogen; $\mathrm{Cr}$, creatinine; UA, uric acid.

no apparent predisposition of either sex (1). IM usually runs a self-limiting course. The majority of IM patients recover without sequelae and return to normal activities $\sim 2$ months after the onset. As numerous individuals are EBV-positive, special precautions against transmission are not necessary. However, severe complications (including upper airway obstruction, hemolytic anemia, thrombocytopenia, hepatitis, myocarditis, splenic rupture, neurological and hematological complications) may occur, and fulminant infection is also possible. Clinical experience suggests that corticosteroids are helpful in the management of these complications, although randomized trials evaluating their efficacy are limited $(1,2)$. No specific guidelines are currently available for the treatment of IM, and no serum factor for predicting IM in either sexes has been identified $(1,2)$. The findings of the present study indicate that UA levels may be such a predictor.

There are established theories as to why normal level of UA is important. Humans cannot efficiently catabolize UA to a more soluble compound (allantoin), due to lack of urate oxidase function. This hepatic enzyme is inactivated during early primate evolution due to two independent nonsense mutations (21). As a result, humans naturally have higher levels of UA compared with most non-primates. This genetic modification actually confers an evolutionary advantage. Under conditions of increased oxidative stress, UA may be oxidized into allantoin and other metabolites via non-enzymatic oxidation and through exposure to pro-oxidant molecules (22). UA is the most abundant natural antioxidant in humans and it accounts for two-thirds of the antioxidant capacity of the plasma (23). However, too high a level of UA is also detrimental, as it exerts a pro-oxidant effect. In the clinical setting, higher levels of UA have been associated with gout $(24,25)$, and associations between hyperuricemia and an increased risk 
Table III. Comparison of hyperuricemia incidence between sexes.

Incidence (case number count) in different sexes

\begin{tabular}{|c|c|c|c|c|}
\hline \multirow{2}{*}{$\begin{array}{l}\text { Incidence } \\
\text { comparisons }\end{array}$} & \multicolumn{2}{|c|}{ Men } & \multicolumn{2}{|c|}{ Women } \\
\hline & IM & Control & IM & Control \\
\hline Normal UA & $89.36 \%(42)$ & $78.72 \%(37)$ & $100.00 \%$ & $87.50 \%(42)$ \\
\hline Hyperuricemia $^{a}$ & $10.64 \%(5)$ & $21.28 \%(10)$ & $0.00 \%(0)$ & $12.50 \%(6)$ \\
\hline Chi-squared value ${ }^{\mathrm{b}}$ & 1.983 & & $6.400^{\mathrm{c}}$ & \\
\hline
\end{tabular}

${ }^{\mathrm{a}}$ Hyperuricemia was defined as UA $>420 \mu \mathrm{mol} / \mathrm{l}$ in men, and $>360 \mu \mathrm{mol} / 1$ in women. ${ }^{\mathrm{b}}$ The incidence of hyperuricemia was compared between men and women by the Chi-squared method. ${ }^{c} \mathrm{P}<0.05$. IM, infectious mononucleosis; UA, uric acid.

Table IV. Pearson's bivariate correlations between UA and other variables in the two sexes.

\begin{tabular}{lcc}
\hline & \multicolumn{2}{c}{ Correlation coefficients } \\
\cline { 2 - 3 } Parameters & Men & Women \\
\hline Age & -0.069 & -0.069 \\
BMI & $0.492^{\mathrm{b}}$ & 0.195 \\
WBC & 0.112 & 0.127 \\
RBC & $0.419^{\mathrm{b}}$ & $0.474^{\mathrm{b}}$ \\
Hb & $0.388^{\mathrm{b}}$ & $0.445^{\mathrm{b}}$ \\
PLT & 0.118 & 0.065 \\
ALT & 0.060 & -0.166 \\
AST & -0.015 & -0.132 \\
TB & $0.225^{\mathrm{a}}$ & 0.196 \\
BUN & $0.247^{\mathrm{a}}$ & $0.506^{\mathrm{b}}$ \\
Cr & $0.452^{\mathrm{b}}$ & $0.496^{\mathrm{b}}$ \\
\hline
\end{tabular}

${ }^{\mathrm{a}} \mathrm{P}<0.05$. ${ }^{\mathrm{b}} \mathrm{P}<0.01$. UA, uric acid; $\mathrm{BMI}$, body mass index; WBC, white blood cell; RBC, red blood cell; Hb, hemoglobin; PLT, platelet; ALT, alanine aminotransferase; AST, aspartate aminotransferase; TB, total bilirubin; BUN, blood urea nitrogen; $\mathrm{Cr}$, creatinine.

of various metabolic disorders have also been described $(5,6)$. In fact, a U-shaped association between extremely low or high UA levels and worse outcome has been described in stroke $(10,26)$. However, it appears that, under conditions of increased oxidative stress, as occurs in acute ischemic stroke, the balance between anti- and pro-oxidant properties shifts to promote neuroprotection (27-30).

In ischemic stroke, highly reactive oxidant molecules are the major force driving the ischemic cascade (31). The brain develops enzymatic and non-enzymatic endogenous antioxidant defenses. UA, being a non-enzymatic molecule, is a powerful antioxidant at physiological concentrations. It was observed that a gradual depletion of UA occurred during the acute phase of stroke (32). Moreover, decreases in UA after stroke onset have been correlated with increased severity and poor long-term outcome (33). Based on promising pre-clinical evidence $(32,34)$, more clinical trials of exogenous administration of UA for stroke are currently performed (8). The mechanisms underlying the role of UA in MS have also been extensively investigated. It has been observed that MS and gout are mutually exclusive (35). It is now generally accepted that the lower serum UA level in MS patients may be due to the intrinsically reduced antioxidant capacity, as well as the increased consumption of UA in MS $(11,12)$. The mechanisms of CNS injury during infection are complex. It has been indicated that oxidative stress and antioxidant imbalance play a central role in the pathophysiology of meningitis (36-38). Recently, Liu et al (13) reported that the serum levels of UA in patients with various types of CNS infections were significantly lower compared with those in normal subjects. However, after effective therapy, the UA levels increased significantly compared with prior to treatment, and were almost restored to normal in some patients.

The design of the present study framework focused on EBV infection causing IM. In fact, it is known that increased oxidative stress plays a fundamental role in the pathogenesis of several types of infections, causing extensive cellular and tissue damage. Previous studies have demonstrated that this mechanism exists in various pathogens, including influenza virus (39), hepatitis virus (40), respiratory viruses (41), human immunodeficiency virus (42), Staphylococcus aureus (43), Helicobacter pylori (44), spirochetal bacteria (45) and mycoplasma (46), among others. It would be reasonable to deduce that infection due to EBV may also cause oxidative stress, leading to obvious depletion of antioxidants, such as UA. In addition, three early clinical studies demonstrated a transitory UA increase during acute onset of IM, which was explained by the increase in de novo purine biosynthesis necessary to accommodate the stepped-up nucleic acid production in IM (16-18). In fact, IM patients visiting our hospital (a tertiary hospital in Tianjin Municipality with a population of 20 million) were often cases with more severe complications, with an IM disease duration of $>10-14$ days. In such patients, oxidative stress and depletion of the antioxidants may well overwhelm the de novo purine biosynthesis of UA. Therefore, this may be considered as the mechanism underlying the findings of the present study.

However, the reason for the obvious female predisposition to IM under conditions of low UA levels remains unclear. It is a common phenomenon that men have a significantly higher level of serum UA compared with women, and the rate of increase in UA levels is also significantly higher in men (5). 
$\mathbf{A}$

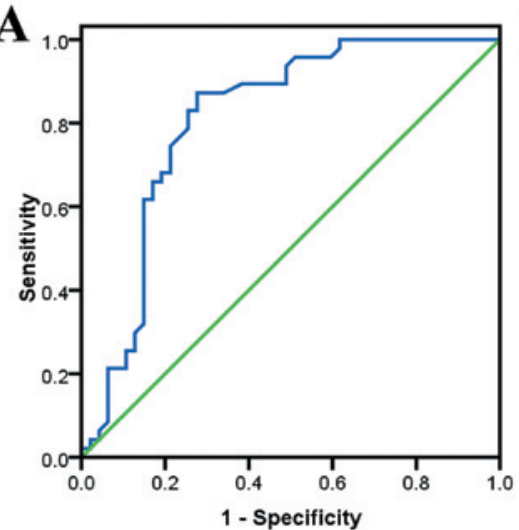

B

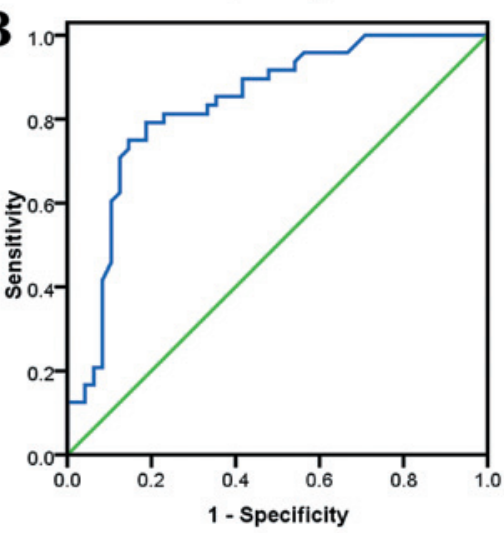

Figure 1. Diagnostic values of uric acid for infectious mononucleosis in (A) male and (B) female patients analyzed by receiver operating characteristic curves.

The present study also confirmed this finding (Table III). A higher level of UA may promote a stronger antioxidant protection in men. Thus, women may be more vulnerable to oxidative stress-related UA depletion, which was also demonstrated by our findings. As a result, a decreased UA level may be more predictive of IM in women (Fig. 1, Table V).

There were certain limitations to the present study. First, the cross-sectional nature of the investigation meant that no causality could be determined from the results. A prospective study should be planned in the future. Second, a limited number of IM patients and controls were included. More participants should be recruited in order to limit the case number-related inherent drawback. Third, due to study budget limitations, measurements such as reactive oxygen species and activities of antioxidants were not performed, which should be included in future investigations. Finally, administration of UA as an adjuvant therapy should be investigated in the future to validate the findings of the present study.

To the best of our knowledge, this is the first study to demonstrate the inverse association between UA and IM, suggesting a progressive decrease of antioxidant reserve in IM. Moreover, low UA level is predictive for IM, particularly in women.

\section{Acknowledgements}

The present study was supported by the National Key Clinical Specialty Project, awarded to the Departments of Nuclear Medicine and Radiology; the Tianjin Medical University General Hospital New Century Excellent Talent Program, Young and 
Middle-aged Innovative Talent Training Program from Tianjin Education Committee, and Talent Fostering Program (the 131 Project) from the Tianjin Education Committee, Tianjin Human Resources and Social Security Bureau, awarded to Zhaowei Meng; the China National Natural Science Foundation (grant no. 81571709), Key Project of Tianjin Science and Technology Committee Foundation (grant no. 16JCZDJC34300), awarded to Zhaowei Meng; and the Tianjin Science and Technology Committee Foundation (grant nos. 11ZCGYSY05700, 12ZCZDSY20400 and 13ZCZDSY20200) awarded to Qing Zhang, Qiyu Jia and Kun Song.

\section{References}

1. Luzuriaga K and Sullivan JL: Infectious mononucleosis. N Engl J Med 362: 1993-2000, 2010

2. Vouloumanou EK, Rafailidis PI and Falagas ME: Current diagnosis and management of infectious mononucleosis. Curr Opin Hematol 19: 14-20, 2012.

3. Lennon P, O' Neill JP, Fenton JE and O' Dwyer T: Challenging the use of the lymphocyte to white cell count ratio in the diagnosis of infectious mononucleosis by analysis of a large cohort of monospot test results. Clin Otolaryngol 35: 397-401, 2010.

4. Henle G, Henle W and Diehl V: Relation of Burkitt's tumor-associated herpes-ytpe virus to infectious mononucleosis. Proc Natl Acad Sci USA 59: 94-101, 1968.

5. Zhang Q, Lou S, Meng Z and Ren X: Gender and age impacts on the correlations between hyperuricemia and metabolic syndrome in Chinese. Clin Rheumatol 30: 777-787, 2011.

6. Zhang J, Meng Z, Zhang Q, Liu L, Song K, Tan J, Li X, Jia Q, Zhang $\mathrm{G}$ and He Y: Gender impact on the correlations between subclinical thyroid dysfunction and hyperuricemia in Chinese. Clin Rheumatol 35: 143-149, 2016.

7. Liu L, Lou S, Xu K, Meng Z, Zhang Q and Song K: Relationship between lifestyle choices and hyperuricemia in Chinese men and women. Clin Rheumatol 32: 233-239, 2013.

8. Llull L, Amaro S and Chamorro Á: Administration of uric acid in the emergency treatment of acute ischemic stroke. Curr Neurol Neurosci Rep 16: 4, 2016.

9. Wang Z, Lin Y, Liu Y, Chen Y, Wang B, Li C, Yan S, Wang Y and Zhao W: Serum uric acid levels and outcomes after acute ischemic stroke. Mol Neurobiol 53: 1753-1759, 2016

10. Zhang X, Huang ZC, Lu TS, You SJ, Cao YJ and Liu CF: Prognostic significance of uric acid levels in ischemic stroke patients. Neurotox Res 29: 10-20, 2016

11. Liu B, Shen Y, Xiao K, Tang Y, Cen L and Wei J: Serum uric acid levels in patients with multiple sclerosis: A meta-analysis. Neurol Res 34: 163-171, 2012

12. Moccia M, Lanzillo R, Costabile T, Russo C, Carotenuto A, Sasso G, Postiglione E, De Luca Picione C, Vastola M, Maniscalco GT, et al: Uric acid in relapsing-remitting multiple sclerosis: A 2-year longitudinal study. J Neurol 262: 961-967, 2015.

13. Liu J, Li M, Wang X, Yi H, Xu L, Zhong XF and Peng FH: Serum uric acid levels in patients with infections of central nervous system. Acta Neurol Belg 116: 303-308, 2016.

14. Collazos J, Blanco MS, Guerra E, Mayo J and Martínez E: Sequential evaluation of serum urate concentrations in AIDS patients with infections of the central nervous system. Clin Chem Lab Med 38: 1293-1296, 2000.

15. Morato-Conceicao YT, Alves-Junior ER, Arruda TA, Lopes JC and Fontes CJ: Serum uric acid levels during leprosy reaction episodes. PeerJ 4: e1799, 2016.

16. Dylewski JS and Gerson M: Hyperuricemia in patients with infectious mononucleosis. Can Med Assoc J 132: 1169-1170, 1985.

17. Cowdrey SC: Hyperuricemia in infectious mononucleosis. JAMA 196: 319-321, 1966.

18. Cowdrey SC: Hyperuricemia in infectious mononucleosis: Further observations. J Am Coll Health Assoc 18: 382-383, 1970

19. Sugita K, Hagisawa S, Satoh Y, Eguchi M and Furukawa T: Recurrent hepatosplenomegaly and peripheral blood cytopenia persistent epstein-barr virus infection and central nervous system manifestation in a patient with lymphadenopathy and low serum uric acid. Acta Paediatr Jpn 40: 362-366, 1998.

20. Moccia M, Lanzillo R, Palladino R, Russo C, Carotenuto A, Massarelli M, Vacca G, Vacchiano V, Nardone A, Triassi M and Morra VB: Uric acid: A potential biomarker of multiple sclerosis and of its disability. Clin Chem Lab Med 53: 753-759, 2015.
21. Wu XW, Muzny DM, Lee CC and Caskey CT: Two independent mutational events in the loss of urate oxidase during hominoid evolution. J Mol Evol 34: 78-84, 1992.

22. Santos CX, Anjos EI and Augusto O: Uric acid oxidation by peroxynitrite: Multiple reactions, free radical formation and amplification of lipid oxidation. Arch Biochem Biophys 372: 285-294, 1999.

23. Becker BF: Towards the physiological function of uric acid. Free Radic Biol Med 14: 615-631, 1993.

24. Messerli FH, Makani H and Halpern D: Gout. N Engl J Med 364: 1876-1877, 2011.

25. Neogi T: Clinical practice. gout. N Engl J Med 364: 443-452, 2011

26. Kanellis J and Kang DH: Uric acid as a mediator of endothelial dysfunction, inflammation and vascular disease. Semin Nephrol 25: 39-42, 2005.

27. Sautin YY and Johnson RJ: Uric acid: The oxidant-antioxidant paradox. Nucleosides Nucleotides Nucleic Acids 27: 608-619, 2008

28. Proctor PH: Uric acid: Neuroprotective or neurotoxic? Stroke 39: e88, 2008.

29. Dawson J, Quinn T, Lees K and Walters M: The continued yin and yang of uric acid. Stroke 39: e9, 2008.

30. Proctor PH: Uric acid and neuroprotection. Stroke 39: e126, 2008.

31. Lipton P: Ischemic cell death in brain neurons. Physiol Rev 79: 1431-1568, 1999

32. Amaro S, Soy D, Obach V, Cervera A, Planas AM and Chamorro A: A pilot study of dual treatment with recombinant tissue plasminogen activator and uric acid in acute ischemic stroke. Stroke 38: 2173-2175, 2007

33. Brouns R, Wauters A, Van De Vijver G, De Surgeloose D, Sheorajpanday R and De Deyn PP: Decrease in uric acid in acute ischemic stroke correlates with stroke severity, evolution and outcome. Clin Chem Lab Med 48: 383-390, 2010

34. Onetti Y, Dantas AP, Pérez B, Cugota R, Chamorro A, Planas AM, Vila $\mathrm{E}$ and Jiménez-Altayó F: Middle cerebral artery remodeling following transient brain ischemia is linked to early postischemic hyperemia: A target of uric acid treatment. Am J Physiol Heart Circ Physiol 308: H862-H874, 2015.

35. Hooper DC, Spitsin S, Kean RB, Champion JM, Dickson GM, Chaudhry I and Koprowski H: Uric acid, a natural scavenger of peroxynitrite, in experimental allergic encephalomyelitis and multiple sclerosis. Proc Natl Acad Sci USA 95: 675-680, 1998.

36. Nudelman Y and Tunkel AR: Bacterial meningitis: Epidemiology, pathogenesis and management update. Drugs 69: 2577-2596, 2009.

37. Liechti FD, Grandgirard D and Leib SL: Bacterial meningitis: Insights into pathogenesis and evaluation of new treatment options: A perspective from experimental studies. Future Microbiol 10: 1195-1213, 2015.

38. Aycicek A, Iscan A, Erel O, Akcali M and Ocak AR: Oxidant and antioxidant parameters in the treatment of meningitis. Pediatr Neurol 37: 117-120, 2007

39. Checconi P, Salzano S, Bowler L, Mullen L, Mengozzi M, Hanschmann EM, Lillig CH, Sgarbanti R, Panella S, Nencioni L, et al: Redox proteomics of the inflammatory secretome identifies a common set of redoxins and other glutathionylated proteins released in inflammation, influenza virus infection and oxidative stress. PLoS One 10: e0127086, 2015.

40. Zuwała-Jagiełło J, Warwas $M$ and Pazgan-Simon M: Ischemia-modified albumin (IMA) is increased in patients with chronic hepatitis $\mathrm{C}$ infection and related to markers of oxidative stress and inflammation. Acta Biochim Pol 59: 661-667, 2012

41. Komaravelli N and Casola A: Respiratory Viral Infections and subversion of cellular antioxidant defenses. J Pharmacogenomics Pharmacoproteomics 5: 1000141, 2014.

42. Ngondi JL, Oben J, Forkah DM, Etame LH and Mbanya D: The effect of different combination therapies on oxidative stress markers in HIV infected patients in Cameroon. AIDS Res Ther 3: 19, 2006.

43. Chakraborty SP, Das S, Chattopadhyay S, Tripathy S, Dash SK, Pramanik P and Roy S: Staphylococcus aureus infection induced redox signaling and DNA fragmentation in T-lymphocytes: Possible ameliorative role of nanoconjugated vancomycin. Toxicol Mech Methods 22: 193-204, 2012.

44. Aslan M, Nazligul Y, Horoz M, Bolukbas C, Bolukbas FF, Aksoy N, Celik H and Erel O: Serum prolidase activity and oxidative status in Helicobacter pylori infection. Clin Biochem 40: 37-40, 2007.

45. Hébert-Schuster M, Borderie D, Grange PA, Lemarechal H, Kavian-Tessler N, Batteux F and Dupin N: Oxidative stress markers are increased since early stages of infection in syphilitic patients. Arch Dermatol Res 304: 689-697, 2012

46. Kariya C, Chu HW, Huang J, Leitner H, Martin RJ and Day BJ: Mycoplasma pneumoniae infection and environmental tobacco smoke inhibit lung glutathione adaptive responses and increase oxidative stress. Infect Immun 76: 4455-4462, 2008. 